\title{
Asynchronous Sampling for Decentralized Periodic Event-Triggered Control
}

\author{
Anqi Fu, Ivana Tomić, and Julie A. McCann
}

\begin{abstract}
Decentralized periodic event-triggered control (DPETC) strategies are an attractive solution for wireless cyberphysical systems where resources such as network bandwidth and sensor power are scarce. This is because these strategies have the advantage of preventing unnecessary data transmissions and therefore reduce bandwidth and energy requirements, however the sensor sampling regime remains synchronous. Typically the action of sampling leads almost immediately to a transmission on an event being detected. If the sampling is synchronous, multiple transmission requests may be raised at the same time which further leads to bursty traffic patterns. Bursty traffic patterns are critical to the DPETC systems performance as the probability of collisions and the amount of requested bandwidth resources become high ultimately causing delays. In this paper, we propose an asynchronous sampling scheme for DPETC. The scheme ensures that at each sampling time, no more than one transmission request can be generated which prevents the occurrence of network traffic collision. At the same time, for the DPETC system with asynchronous sampling a pre-designed global exponential stability and $\mathcal{L}_{2}$ gain performance can still be guaranteed. We illustrate the effectiveness of the approach through a numerical example.
\end{abstract}

\section{INTRODUCTION}

Cyber-physical systems (CPSs) are a tight integration of sensing, control, communication, computation and physical processes. Application of wireless sensors and actuators in a variety of domains, including civil infrastructure, energy, healthcare, manufacturing, and transportation offers many benefits. The absence of wires for communication reduces their deployment costs, maintenance effort, and provides greater flexibility for sensor location and system architecture.

The challenges that accompany these benefits are twofold. The first challenge is that the effects of the inherent non-determinism of wireless channel behaviour contradicts the hard real-time requirements typical of control system. The amount of transmission delay or data loss from the wireless channel that the controller can tolerate has to be bounded. Otherwise, the system stability or performance guarantees may be lost. The second challenge is that the bandwidth of the wireless networks and their source of power (batteries or low-power energy harvesters) are highly constrained. Therefore, the number of data transmissions and control task executions have to be significantly low to maintain the operation of the sensor network, but necessary

This work has been funded by Alan Turing Institute as part of the Data Centric Engineering (DCE) Programme, UK EPSRC as part of the Science of Sensor System Software (S4) programme grant no. EP/N007565/1, and China Scholarship Council (CSC).

A. Fu, I. Tomić, and J. McCann are with the Department of Computing, Imperial College London, London SW7 2AZ, UK. Email: a.fu, i.tomic, j.mccanneimperial.ac.uk. to close the loop while maintaining system stability and performance. Also, the amount of time that sensor nodes have their radios on listening for possible communications from other nodes should be minimized as for many sensor device implementations listening consumes the same amount of resources as send or receive.

To mitigate these previously defined challenges in CPSs, event-triggered control has been proposed. Unlike traditional periodic time-triggered control approaches, that transmit data every fixed period of time (even if there is no change in the underlying physical process), event-triggered control close feedback loops only when necessary. Consequently, eventbased communication saves considerable levels of energy lengthening the operational lifetime of the sensor device and lowers the bandwidth requirements. There is an extensive literature on centralized event-triggered control, e.g. [6], [16], [20]. These approaches evolved into decentralized eventtriggered control (e.g. [3]-[5], [12], [13], [17], [19]) which makes them applicable to large-scale CPSs where sensor and actuator nodes are not physically collocated and span over multi-km distances. The work presented in [10] and [7] further refined the existing designs by introducing a decentralized periodic event-triggered control (DPETC) scheme that does not require sensors to continuously monitor the systems' outputs. The scheme uses only local information and it allows the update of the controller output via same eventtriggered mechanism. This is necessary when the controller and actuators are not collocated.

The feasibility of using DPETC has been proved experimentally in [11]. The challenge of DPETC is that the system outputs are sampled synchronously during a regular sample period. This may lead to multiple data transmission requests being initiated at the same time which results in bursty traffic patterns and transmission collisions. The work in [11] uses a Time-Division Multiple Access (TDMA) protocol for channel access where each sensor is allocated a fixed time slot in which to transmit its signal. This way, transmission collisions are avoided, but it requires that all sensor nodes reserve a time slot in each hyperframe of the TDMA, which leads to high wireless bandwidth requirements.

In this work, we design an asynchronous sampling mechanism for DPETC. The mechanism follows a pre-designed schedule which repeats periodically along the system timeline. At each sampling time, only one element of the system's output vector (that includes both, the plant's and the controller's outputs) will be sampled and updated. That means that no more than one transmission request will be generated at each sampling instance. Therefore, elements of the output 
vector are sampled asynchronously. The intervals between any two consecutive samplings of an individual output vector element are equal.

Asynchronous sampling problem has been considered in [14], [9] and [8]. The work in [14] proposes aperiodic sampling intervals for time-triggered control only, therefore is outside the scope of this paper. The work in [9] and [8] proposes a delay-based method for event-triggered control with asynchronous sampling. The method uses the state feedback control which limits the application scope only to a case when all states are available for the feedback. Additionally, it fails to include the controller output update with the eventtriggered mechanism, which makes it unsuitable for systems whose actuators are also distributed and have to communicate with the controller via the same wireless network. Our work has developed a new scheme which applies a hybrid system method and overcomes shortcomings of the existing state of the art. It allows us to analyse more generalized classes of systems that use the output feedback. Also, the applied output feedback controllers can have their own dynamics and update their outputs based on the event-triggered mechanism which allows their distribution over multi-km distances.

This paper is organized as follows. Section II presents the necessary notations and definitions to model our systems. Section III describes the architecture of a particular cyberphysical system, we briefly review DPETC based on [10], and formally provide the problem definition. The main result is presented in Section IV. We demonstrate the feasibility of the approach through a numerical example in Section $\mathrm{V}$ and conclude the work in Section VI.

\section{NOTATION AND PRELIMINARIES}

We denote the $n$-dimensional Euclidean space by $\mathbb{R}^{n}$. We use $\mathbb{R}^{+}$and $\mathbb{R}_{0}^{+}=\mathbb{R}^{+} \cup\{0\}$ to denote the positive real numbers. We use $\mathbb{N}$ to denote the natural numbers including zero, while we use $\mathbb{N}^{+}$to denote the set of natural numbers without including zero. The usual Euclidean $\left(l_{2}\right)$ vector norm is denoted by $|\cdot|$. A symmetric matrix $M \in \mathbb{R}^{n \times n}$ is said to be positive definite, which we denote by $M \succ 0$, if $x^{\mathrm{T}} M x>$ 0 for all $x \neq 0, x \in \mathbb{R}^{n}$. For a symmetric matrix $M \in$ $\mathbb{R}^{n \times n}, \lambda_{\min }(M)$ and $\lambda_{\max }(M)$ denote the minimum and maximum eigenvalue of $M$, respectively. For a matrix $N \in$ $\mathbb{R}^{m \times n}$, we denote by $N^{\mathrm{T}} \in \mathbb{R}^{n \times m}$ the transposed matrix of $N$. For a locally integrable signal $w: \mathbb{R}_{0}^{+} \rightarrow \mathbb{R}^{n}$, we denote by $\|w\|_{\mathcal{L}_{2}}=\sqrt{\int_{0}^{\infty}|w(t)|^{2} \mathrm{~d} t}$ its $\mathcal{L}_{2}$-norm, provided the integral is finite. Furthermore, we define the set of all locally integrable signals with a finite $\mathcal{L}_{2}$-norm as $\mathcal{L}_{2}$. For the sake of brevity, $\left[\begin{array}{cc}A & B \\ B^{\mathrm{T}} & C\end{array}\right]$ sometimes is written as $\left[\begin{array}{cc}A & B \\ * & C\end{array}\right]$.

The notions of exponential stability and $\mathcal{L}_{2}$-gain will be central to our later discussion. These are defined next.

Definition II.1. (Exponential Stability) [15] A system $\dot{\xi}(t)=$ $f(\xi(t)), t \in \mathbb{R}_{0}^{+}, \xi(t) \in \mathbb{R}^{n}$ is said to be globally exponentially stable (GES), if there exist $a, c \in \mathbb{R}^{+}$that for any $t_{0} \geq 0$ satisfy $|\xi(t, \xi(0))| \leq c|\xi(0)| e^{-a t}, \forall t \geq t_{0}$.

Definition II.2. ( $\mathcal{L}_{2}$-gain) [10] The system $\dot{\xi}(t)=$

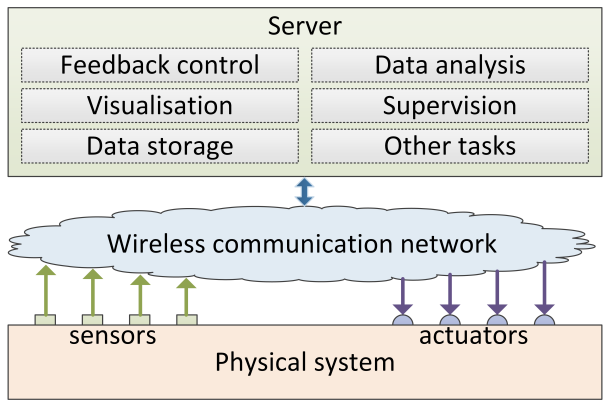

Fig. 1. Cyber-physical system architecture.

$f(\xi(t), w(t)), z(t)=g(\xi(t), w(t))$ is said to have an $\mathcal{L}_{2^{-}}$ gain from $w$ to $z$ smaller than or equal to $\gamma$, if there exists a $\mathcal{K}_{\infty}$ function $\delta: \mathbb{R}^{n_{\xi}} \rightarrow \mathbb{R}^{+}$such that for any $w \in \mathcal{L}_{2}$, any initial state $\xi(0)=\xi_{0} \in \mathbb{R}^{n_{\xi}}$, the corresponding solution to the system satisfies $\|z\|_{\mathcal{L}_{2}} \leq \delta\left(\xi_{0}\right)+\gamma\|w\|_{\mathcal{L}_{2}}$.

\section{PROBLEM DEFINITION}

\section{A. Cyber-Physical System Architecture}

In Fig. 1 we present a diagram of the CPS considered in this paper. The CPS consists of a large complex physical process (the plant) and the server which are connected via a wireless communication network. The physical process is monitored and its outputs are measured by various sensors. Due to a large geographical scale of the physical process, the sensors are non-collocated. We assume that all sensors are battery powered (but much of the work is directly applicable to sensor networks using harvested power). We assume sensor-to-server communication is achieved in a single-hop fashion via a wireless network which is far more favourable for control-based systems due to its increased reliability and lower communication delays compared to multi-hop networks. The server acts as a central node and it executes allocated tasks to ensure the normal operation of the CPS. Similarly, server-to-actuator communication is carried out in a single-hop fashion via the same wireless network. The actuators are also non-collocated and assumed to have unlimited energy supply, from either wired power network or local power generators. We assume that the CPS has a single feedback control loop which is used to ensure the stability or certain level of performance of the physical process.

In this paper, we focus on the feedback control task of the CPS depicted in Fig. 1. Next, we present the mathematical description of the plant and the controller.

The plant is a linear time-invariant (LTI) system given by

$$
\left\{\begin{aligned}
\dot{\xi}_{p}(t) & =A_{p} \xi_{p}(t)+B_{p} \hat{v}(t)+E w(t) \\
y(t) & =C_{p} \xi_{p}(t)
\end{aligned}\right.
$$

where $\xi_{p}(t) \in \mathbb{R}^{n_{p}}$ denotes the state vector of the plant, $\hat{v}(t) \in \mathbb{R}^{n_{v}}$ denotes the input vector of the plant, $y(t) \in$ $\mathbb{R}^{n_{y}}$ denotes the output vector of the plant at time $t$. The vector $w(t) \in \mathbb{R}^{n_{w}}$ denotes an unknown disturbance to the system at time $t . A_{p}, B_{p}, C_{p}$ and $E$ are the matrices chosen appropriately. The matrix $E$ represents the distribution of the disturbance. 
The plant is controlled by a digital controller given by

$$
\left\{\begin{aligned}
\xi_{c}\left(t_{k+1}\right) & =A_{c} \xi_{c}\left(t_{k}\right)+B_{c} \hat{y}\left(t_{k}\right) \\
v\left(t_{k}\right) & =C_{c} \xi_{c}\left(t_{k}\right)+D_{c} \hat{y}\left(t_{k}\right)
\end{aligned}\right.
$$

where $\xi_{c}\left(t_{k}\right) \in \mathbb{R}^{n_{c}}$ is the state vector of the controller, $\hat{y}\left(t_{k}\right) \in \mathbb{R}^{n_{y}}$ is the input vector of the controller, $v\left(t_{k}\right) \in \mathbb{R}^{n_{v}}$ is the output vector of the controller at time $t_{k} . A_{c}, B_{c}, C_{c}$ and $D_{c}$ are the matrices chosen appropriately.

From (2), we can see that the controller has a memory, i.e. its own state. This allows us to compute the controller output, but not necessary update the plant input instantaneously. Instead, the transmissions from the controller to the actuators are triggered by the event-triggering mechanism (similar to the transmissions from the sensors to the controller). The controller works in discrete-time where $t_{k}, k \in \mathbb{N}$ are the sampling times, which are periodic in the sense that $t_{k}=k h$, $k \in \mathbb{N}$ for the chosen constant execution interval $h>0$. The periodic execution sequence of the controller is defined as:

$$
\mathcal{T}_{k}:=\left\{t_{k} \mid t_{k}:=k h, k \in \mathbb{N}\right\} .
$$

We define $\tau: \mathbb{R}_{0}^{+} \rightarrow \mathbb{R}_{0}^{+}$to be the time that elapsed since the last execution time:

$$
\tau(t):=t-t_{k}, t \in\left[t_{k}, t_{k+1}\right] .
$$

For the implementation of the plant in (1) and the controller in (2), we define two vectors:

$$
\hat{u}\left(t_{k}\right):=\left[\begin{array}{l}
\hat{y}\left(t_{k}\right) \\
\hat{v}\left(t_{k}\right)
\end{array}\right] \in \mathbb{R}^{n_{u}}, u(t):=\left[\begin{array}{l}
y(t) \\
v(t)
\end{array}\right] \in \mathbb{R}^{n_{u}} .
$$

$\hat{u}\left(t_{k}\right)$ is the input of the implementation, $u(t)$ is the output of the implementation, while $n_{u}:=n_{y}+n_{v}$. The input $\hat{u}\left(t_{k}\right)$ is sampled and updated from $u(t)$ by DPETC which will be discussed in Subsection III-B. The updated value of the input $\hat{u}\left(t_{k}\right)$ is held until next sampling time based on a zero-order hold $(\mathrm{ZOH})$ mechanism.

We define a performance output of the implementation, $z \in \mathbb{R}_{n_{z}}$, as

$$
z(t):=\bar{C} \xi(t)+\bar{D} w(t)
$$

where

$$
\xi(t):=\left[\begin{array}{llll}
\xi_{p}^{\mathrm{T}}(t) & \xi_{c}^{\mathrm{T}}(t) & \hat{y}^{\mathrm{T}}(t) & \hat{v}^{\mathrm{T}}(t)
\end{array}\right]^{\mathrm{T}} \in \mathbb{R}^{n_{\xi}},
$$

and $n_{\xi}:=n_{p}+n_{c}+n_{u} \cdot w(t)$ has been defined in (1). $\bar{C}$ and $\bar{D}$ are the matrices chosen appropriately.

\section{B. Decentralized Periodic Event-Triggered Control}

As discussed in Section I, DPETC reduces communication requirements in CPSs when compared to centralized eventtriggered control schemes or more traditional time-triggered control schemes. In this section, we give a brief overview of DPETC. For more details, we refer the reader to [10].

Now define $n_{u}$ sample-and-update sequences as:

$$
\mathcal{T}_{b_{i}}^{i}:=\left\{t_{b_{i}}^{i} \mid b_{i} \in \mathbb{N}\right\} \subseteq \mathcal{T}_{k}, i \in\left\{1, \cdots, n_{u}\right\} .
$$

In each sequence $\mathcal{T}_{b_{i}}^{i}$, the sample-and-update time $t_{b_{i}}^{i}$ is determined by:

$$
t_{b_{i}}^{i}:=\left\{t \in \mathcal{T}_{k}, t>t_{b_{i}-1}^{i} \mid \xi^{\mathrm{T}}(t) Q_{i} \xi(t)>0\right\}
$$

where the matrix $Q_{i}$ is defined as

$$
\begin{aligned}
& Q_{i}:= \\
& {\left[\begin{array}{cc}
\left(1-\sigma_{i}\right) C^{\mathrm{T}} \Gamma_{i} C & \left(1-\sigma_{i}\right) C^{\mathrm{T}} \Gamma_{i} D-C^{\mathrm{T}} \Gamma_{i} \\
* & (D-I)^{\mathrm{T}} \Gamma_{i}(D-I)-\sigma_{i} D^{\mathrm{T}} \Gamma_{i} D
\end{array}\right]}
\end{aligned}
$$

with $I$ being an identity matrix of appropriate dimension. The matrix $\Gamma_{i}$ is defined as

$$
\Gamma_{i}=\operatorname{diag}\left\{\gamma_{i}^{1}, \cdots, \gamma_{i}^{n_{u}}\right\}
$$

where $\gamma_{i}^{j}=1$ when $i=j$, otherwise $\gamma_{i}^{j}=0$. Furthermore, $\sigma_{i}, i \in\left\{1, \cdots, n_{u}\right\}$ represent a set of scalar numbers and the matrices $C$ and $D$ are

$$
C:=\left[\begin{array}{cc}
C_{p} & \mathbf{0} \\
\mathbf{0} & C_{c}
\end{array}\right], D:=\left[\begin{array}{cc}
\mathbf{0} & \mathbf{0} \\
D_{c} & \mathbf{0}
\end{array}\right] .
$$

$\mathbf{0}$ is a zero matrix of appropriate dimension. Note that, in this paper $\mathbf{0}$ represents zero matrix, while 0 represents the scalar. The matrix $\Gamma_{i}$ can also be expressed as:

$$
\Gamma_{i}=\operatorname{diag}\left\{\Gamma_{i}^{y}, \Gamma_{i}^{v}\right\},
$$

where $\Gamma_{i}^{y}:=\left\{\gamma_{i}^{1}, \cdots, \gamma_{i}^{n_{y}}\right\}, \Gamma_{i}^{v}:=\left\{\gamma_{i}^{n_{y}+1}, \cdots, \gamma_{i}^{n_{u}}\right\}$. Based on (5) we define

$$
\begin{aligned}
\hat{u}\left(t_{k}\right) & =\left[\begin{array}{lll}
\hat{u}_{1}\left(t_{k}\right) & \cdots & \hat{u}_{n_{u}}\left(t_{k}\right)
\end{array}\right]^{\mathrm{T}} \\
u(t) & =\left[\begin{array}{lll}
u_{1}(t) & \cdots & u_{n_{u}}(t)
\end{array}\right]^{\mathrm{T}},
\end{aligned}
$$

as the input and the output of the implementation. At each sampling time $t_{k}$, the input applied to the implementation, $\hat{u}\left(t_{k}\right)$, is updated as $\forall t_{k} \in \mathcal{T}_{b_{i}}^{i}$ :

$$
\hat{u}_{i}\left(t_{k}\right)= \begin{cases}u_{i}\left(t_{k}\right), & \text { if } t_{k} \in \mathcal{T}_{b_{i}}^{i} \\ \hat{u}_{i}\left(t_{k-1}\right), & \text { otherwise. }\end{cases}
$$

Then, the error caused by the sample-and-hold mechanism can be defined as

$$
e_{i}(t):=\hat{u}_{i}\left(t_{k}\right)-u_{i}(t), t \in\left[t_{k}, t_{k+1}\right) .
$$

We can observe from (9), (10), and (16) that the eventtriggering condition is based on the relation between the local sample-and-hold error $e_{i}(t)$ and the local current implementation output $u_{i}(t)$. Therefore, the design is decentralized as only local information is used for its calculation.

From (9), one can easily find that there may be more than one element of the input $\hat{u}\left(t_{k}\right)$ to be updated at the same sampling time $t_{k}$. We define the set $\mathcal{J} \subseteq \overline{\mathcal{J}}:=$ $\left\{1, \cdots, n_{u}\right\}$ that indicates the occurrence of an event $i$ in $u(t)=\left[\begin{array}{lll}u_{1}(t) & \cdots & u_{n_{u}}(t)\end{array}\right]^{\mathrm{T}}$ where $i=1, \cdots, n_{u}$. The set $\mathcal{J}$ is defined at each sampling time, $t_{k}$. Similarly, $\mathcal{J}_{c}:=$ $\overline{\mathcal{J}} \backslash \mathcal{J}$. We can reformulate the event-triggered condition in (9) as a set of memberships where

$$
\begin{array}{ll}
i \in \mathcal{J}, & \text { iff } \xi^{\mathrm{T}}\left(t_{k}\right) Q_{i} \xi\left(t_{k}\right)>0 \\
i \in \mathcal{J}_{c}, & \text { otherwise. }
\end{array}
$$

According to [10], the event-triggering mechanism (9) and (15) can render the system (1), (2), and (6) GES with a predesigned decay rate when $w=0$, and has an $\mathcal{L}_{2}$-gain from $w$ to $z$ smaller than or equal to a pre-designed value if $w \in \mathcal{L}_{2}$. 


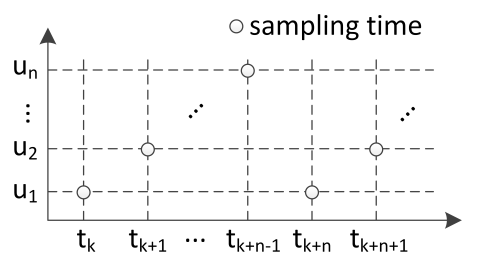

Fig. 2. An example of the asynchronous sampling schedule where the sequence is determined by ordering sensors' labels in an ascending order.

TDMA hyperframe

\begin{tabular}{|l|l|l|l|l|l|l|l|l|l|}
\hline $\mathrm{u}_{1}$ & $\mathrm{u}_{2}$ & $\ldots$ & $\mathrm{u}_{\mathrm{n}}$ & Other tasks & $\mathrm{u}_{1}$ & $\mathrm{u}_{2}$ & $\ldots$ & $\mathrm{u}_{\mathrm{n}}$ & Other tasks \\
\hline $\mathrm{u}_{1}$ & \multicolumn{6}{c|}{ Other tasks } & $\mathrm{u}_{2}$ & \multicolumn{3}{|c|}{ Other tasks } \\
\hline
\end{tabular}

Fig. 3. A TDMA hyperframe. The first row depicts the design in [11] where multiple slots are reserved for the feedback control loop. The second row depicts our design with a single slot allocated for the feedback control loop.

\section{Problem Definition}

Following the discussion in the current Section and Section I, we assume the output-feedback controller that deploys DPETC. At the same time we want to avoid bursty traffic patterns that may occur at individual sampling times. In doing so, we aim to extend the DPETC design by applying an asynchronous sampling schedule. An example of the sampling schedule is depicted in Figure 2. The proposed approach reduces the data load on the wireless network which makes it a promising design for future TDMA-based MAC protocols. Unlike the design in [11] where multiple slots are reserved for transmissions, in our design we reserve a single slot for the feedback control loop (see Fig. 3). This potentially may lead to a better channel utility as only one slot per TDMA hyperframe will be reserved for control tasks. Next, we give a formal definition of the problem that is being addressed in this paper.

Problem III.1. Consider the plant (1), controller (2) and the periodic execution sequence (3). Based on DPETC in (9), (10), (15), a decentralized event-triggered control mechanism with asynchronous sampling schedule should be designed, such that at each sampling time $t_{k}$, no more than one transmission requirement is generated. At the same time, a pre-designed GES and $\mathcal{L}_{2}$-gain performance must be guaranteed.

\section{DECENTRALIZED PERIODIC EVENT-TRIGGERED CONTROL WITH ASYNCHRONOUS SAMPLING}

In this section, we present our solution that addresses the Problem III.1 of asynchronous sampling for CPSs that use DPETC.

We define $\mathcal{S}_{O}$ to be a finite sequence containing the sampling schedule of the elements of $u(t)$ in (14). We assume that, in $\mathcal{S}_{o}$, each element $i$, where $i \in\left\{1, \cdots, n_{u}\right\}$ always appears once, and once fixed, the order won't change. We refer to the process of sampling the whole vector of $u(t)$ by following the schedule $\mathcal{S}_{o}$ as a round. We define two mappings: $l: \mathbb{R}_{0}^{+} \rightarrow \mathbb{N}^{+}$and $\kappa: \mathbb{R}_{0}^{+} \rightarrow \mathbb{N}$. $l(t)$ denotes a pointer to $\mathcal{S}_{o}$ that indicates the next sampling element of $u(t)$, i.e. $u_{\mathcal{S}_{o}(l(t))}(t)$ while $\kappa(t)$ denotes a counter of the round.

Following this description, we need to redefine the decentralized event-triggered condition given in (9) as:

$$
t_{b_{i}}^{i}:=\left\{t \in \mathcal{T}_{k}, t>t_{b_{i}-1}^{i}, \mathcal{S}_{o}(l(t))=i \mid \xi^{\mathrm{T}}(t) Q_{i} \xi(t)>0\right\} .
$$

This means that before checking if the triggering mechanism is satisfied for element $i$ at sampling time $t_{k}$, we need to check if the element $i$ is allowed to sample at $t_{k}$.

Similarly, we redefine the sets $\mathcal{J}$ and $\mathcal{J}_{c}$ in (17) as:

$$
\begin{aligned}
& \forall i \in\left\{1, \cdots, n_{u}\right\}, \kappa(t)=\text { a constant number : } \\
& i \in \mathcal{J}, \quad \text { if } \mathcal{S}_{o}(l(t))=i, \xi^{\mathrm{T}}\left(t_{k}\right) Q_{i} \xi\left(t_{k}\right)>0 \\
& i \in \mathcal{J}_{c}, \quad \text { if } \mathcal{S}_{o}(l(t))=i, \xi^{\mathrm{T}}\left(t_{k}\right) Q_{i} \xi\left(t_{k}\right) \leq 0 .
\end{aligned}
$$

As we can see, $\kappa(t)$ is invariant, which means that the membership set in (19) holds for all elements of $u(t)$ that belong to a single round. Within this round, each element of $u(t), u_{i}(t)$, will have its own sampling time. At that time, $i \in \mathcal{J}$ and the quadratic term in (19) is bigger than 0 .

An impulsive system is constructed from (1), (2), (6) and (19), as

$$
\begin{aligned}
& {\left[\begin{array}{l}
\dot{\xi}(t) \\
\dot{\tau}(t) \\
\dot{l}(t) \\
\dot{\kappa}(t)
\end{array}\right]=\left[\begin{array}{c}
\bar{A} \xi(t)+\bar{B} w(t) \\
1 \\
0 \\
0
\end{array}\right], \text { when } \tau(t) \in[0, h)} \\
& {\left[\begin{array}{l}
\xi\left(t^{+}\right) \\
\tau\left(t^{+}\right) \\
l\left(t^{+}\right) \\
\kappa\left(t^{+}\right)
\end{array}\right]=\left[\begin{array}{c}
J_{\mathcal{S}_{o}(l(t))} \xi(t) \\
0 \\
l(t)+1 \\
\kappa(t)
\end{array}\right], \text { when }\left\{\begin{array}{l}
\tau(t)=h \\
l(t)<n_{u} \\
\mathcal{S}_{o}(l(t)) \in \mathcal{J}
\end{array}\right.} \\
& {\left[\begin{array}{l}
\xi\left(t^{+}\right) \\
\tau\left(t^{+}\right) \\
l\left(t^{+}\right) \\
\kappa\left(t^{+}\right)
\end{array}\right]=\left[\begin{array}{c}
J_{\mathcal{S}_{o}(l(t))} \xi(t) \\
0 \\
1 \\
\kappa(t)+1
\end{array}\right], \text { when }\left\{\begin{array}{l}
\tau(t)=h \\
l(t)=n_{u} \\
\mathcal{S}_{o}(l(t)) \in \mathcal{J}
\end{array}\right.} \\
& {\left[\begin{array}{l}
\xi\left(t^{+}\right) \\
\tau\left(t^{+}\right) \\
l\left(t^{+}\right) \\
\kappa\left(t^{+}\right)
\end{array}\right]=\left[\begin{array}{c}
J_{0} \xi(t) \\
0 \\
l(t)+1 \\
\kappa(t)
\end{array}\right], \text { when }\left\{\begin{array}{l}
\tau(t)=h \\
l(t)<n_{u} \\
\mathcal{S}_{o}(l(t)) \in \mathcal{J}_{c}
\end{array}\right.} \\
& {\left[\begin{array}{l}
\xi\left(t^{+}\right) \\
\tau\left(t^{+}\right) \\
l\left(t^{+}\right) \\
\kappa\left(t^{+}\right)
\end{array}\right]=\left[\begin{array}{c}
J_{0} \xi(t) \\
0 \\
z(t) \\
1 \\
\kappa(t)+1
\end{array}\right], \text { when }\left\{\begin{array}{l}
l(t)=n_{u} \\
\mathcal{S}_{o}(l(t)) \in \mathcal{J}_{c}
\end{array}\right.}
\end{aligned}
$$

where

$$
\begin{gathered}
\bar{A}:=\left[\begin{array}{cccc}
A_{p} & \mathbf{0} & \mathbf{0} & B_{p} \\
\mathbf{0} & \mathbf{0} & \mathbf{0} & \mathbf{0} \\
\mathbf{0} & \mathbf{0} & \mathbf{0} & \mathbf{0} \\
\mathbf{0} & \mathbf{0} & \mathbf{0} & \mathbf{0}
\end{array}\right], \bar{B}:=\left[\begin{array}{c}
E \\
\mathbf{0} \\
\mathbf{0} \\
\mathbf{0}
\end{array}\right], \\
J_{i}:=\left[\begin{array}{cccc}
I & \mathbf{0} & \mathbf{0} & \mathbf{0} \\
B_{c} \Gamma_{i}^{y} C_{p} & A_{c} & B_{c}\left(I-\Gamma_{i}^{y}\right) & \mathbf{0} \\
\Gamma_{i}^{y} C_{p} & \mathbf{0} & \left(I-\Gamma_{i}^{y}\right) & \mathbf{0} \\
\mathbf{0} & \Gamma_{i}^{v} C_{c} & \Gamma_{i}^{v} D_{c} & \left(I-\Gamma_{i}^{v}\right)
\end{array}\right],
\end{gathered}
$$




$$
J_{0}:=\left[\begin{array}{cccc}
I & \mathbf{0} & \mathbf{0} & \mathbf{0} \\
\mathbf{0} & A_{c} & B_{c} & \mathbf{0} \\
\mathbf{0} & \mathbf{0} & I & \mathbf{0} \\
\mathbf{0} & \mathbf{0} & \mathbf{0} & I
\end{array}\right] .
$$

For the impulsive system (20), to analyse its stability and $\mathcal{L}_{2}$-gain performance, we consider the following Lyapunov function:

$$
V(\xi(t), \tau(t), l(t))=\xi^{\mathrm{T}} P_{l}(\tau) \xi,
$$

where $P_{i}:[0, h] \rightarrow \mathbb{R}^{n_{\xi} \times n_{\xi}}, i \in\left\{1, \cdots, n_{u}\right\}$ satisfies the following Riccati differential equation:

$$
\frac{\mathrm{d}}{\mathrm{d} t} P_{i}=-\bar{A}^{\mathrm{T}} P_{i}-P_{i} \bar{A}-2 \rho P_{i}-\gamma^{-2} \bar{C}^{\mathrm{T}} \bar{C}-G^{\mathrm{T}} M G .
$$

The matrices $M$ and $G$ are defined as

$$
\begin{aligned}
M & :=\left(I-\gamma^{-2} \bar{D}^{\mathrm{T}} \bar{D}\right)^{-1} \\
G & :=\bar{B}^{\mathrm{T}} P_{i}+\gamma^{-2} \bar{D}^{\mathrm{T}} \bar{C}
\end{aligned}
$$

and $\rho$ and $\gamma$ are designed parameters. From (22), the Hamiltonian matrix and its exponential can be constructed:

$$
H:=\left[\begin{array}{ll}
H_{11} & H_{12} \\
H_{21} & H_{22}
\end{array}\right], F(\tau):=e^{-H \tau}=\left[\begin{array}{ll}
F_{11}(\tau) & F_{12}(\tau) \\
F_{21}(\tau) & F_{22}(\tau)
\end{array}\right] .
$$

where $H_{11}:=\bar{A}+\rho I+\gamma^{-2} \bar{B} M \bar{D}^{\mathrm{T}} \bar{C}, H_{12}:=\bar{B} M \bar{B}^{\mathrm{T}}$, $H_{21}:=-\bar{C}^{\mathrm{T}}\left(\gamma^{2} I-\bar{D} \bar{D}^{\mathrm{T}}\right)^{-1} \bar{C}, H_{22}:=-(\bar{A}+\rho I+$ $\left.\gamma^{-2} \bar{B} M \bar{D}^{\mathrm{T}} \bar{C}\right)^{\mathrm{T}}$.

Assumption IV.1. $F_{11}(\tau)$ is invertible $\forall \tau \in[0, h]$.

Since $F_{11}(0)=I$ and $F_{11}(\tau)$ is continuous, the Assumption IV.1 will always hold for sufficiently small $h$. If Assumption IV. 1 holds, $-F_{11}^{-1}(h) F_{12}(h)$ is positive semidefinite matrix. Then we can define the matrix $\bar{S}$ that satisfies $\bar{S} \bar{S}^{\mathrm{T}}:=-F_{11}^{-1}(h) F_{12}(h)$. Additionally, we define $P_{i h}:=$ $P_{i}(h)$. Next, we present the main result of this paper.

Theorem IV.2. Consider the system (20), event-triggered mechanism (19), and Lyapunov function (21). Also, assume that the Assumption IV.1 holds. Let $\gamma^{2}>\lambda_{\max }\left(\bar{D}^{\mathrm{T}} \bar{D}\right)$, $\rho>0$. If there exist matrices $P_{i h} \succ 0, \forall i \in\left\{1, \cdots, n_{u}\right\}$, scalars $\mu_{\mathcal{J}^{j}}^{i} \geq 0$, and $\mu_{\mathcal{J}^{j}}^{i} \geq 0$, for all $i, j \in\left\{1, \cdots, n_{u}\right\}$, such that the following LMIs hold:

$$
\begin{aligned}
& {\left[\begin{array}{ccc}
P_{i h}-\mu_{\mathcal{J}^{j}}^{i} Q_{j} & J_{i}^{\mathrm{T}} F_{11}^{-\mathrm{T}}(h) P_{i^{+}} \bar{S} & J_{i^{\star}}^{\mathrm{T}} H_{i} \\
* & I-\bar{S}^{\mathrm{T}} P_{i^{+} h} \bar{S} & \mathbf{0} \\
* & * & H_{i}
\end{array}\right] \succ 0,} \\
& {\left[\begin{array}{ccc}
P_{i h}+\mu_{\mathcal{J}_{c}^{j}}^{i} Q_{j} & J_{0}^{\mathrm{T}} F_{11}^{-\mathrm{T}}(h) P_{i^{+} h} \bar{S} & J_{i^{\star}}^{\mathrm{T}} H_{i} \\
* & I-\bar{S}^{\mathrm{T}} P_{i^{+} h} \bar{S} & \boldsymbol{0} \\
* & * & H_{i}
\end{array}\right] \succ 0,}
\end{aligned}
$$

where

$$
\begin{aligned}
& H_{i}:=F_{11}^{-\mathrm{T}}(h) P_{i^{+} h} F_{11}^{-1}(h)+F_{21}(h) F_{11}^{-1}(h) \\
& i^{+}= \begin{cases}i+1, & \text { if } i<n_{u} \\
1, & \text { if } i=n_{u}\end{cases}
\end{aligned}
$$

Then, the impulsive system (20) is GES with the convergence rate $\rho$ when $w=0$; and the $\mathcal{L}_{2}$-gain from $w$ to $z$ is smaller than or equal to $\gamma$.
Proof. The basic idea of the proof is inspired by the proofs of Theorem III.4 in [1] and Theorem III.2 in [10]. The proof consists of three main steps which are presented next.

The first step is to show that the function $V$ satisfies

$$
c_{1}|\xi|^{2} \leq V(\xi, \tau, l) \leq c_{2}|\xi|^{2}
$$

$\forall t \in \mathbb{R}_{0}^{+}$. Here, $c_{1}$ and $c_{2}$ are scalars that satisfy $0<c_{1} \leq c_{2}$. According to [1] and [10], $\forall \tau \in[0, h]$, it holds that $P_{i}(\tau) \succ$ 0 , and

$$
\begin{aligned}
& c_{1}=\min _{i \in\left\{1, \cdots, n_{u}\right\}} \min _{\tau \in[0, h]} \lambda_{\min }\left(P_{i}(\tau)\right) \\
& c_{2}=\max _{i \in\left\{1, \cdots, n_{u}\right\}} \max _{\tau \in[0, h]} \lambda_{\max }\left(P_{i}(\tau)\right) .
\end{aligned}
$$

The second step is to show that $V$ has a supply rate $\gamma^{-2} z^{\mathrm{T}} z-w^{\mathrm{T}} w$ during the flow of the impulsive system (20). Since $\gamma^{2}>\lambda_{\max }\left(\bar{D}^{\mathrm{T}} \bar{D}\right)$ from the hypothesis of the theorem, by derivating $V$ along $t$, the following inequality can be obtained directly for $t \in[0, h]$ :

$$
\frac{\mathrm{d}}{\mathrm{d} t} V(t) \leq-2 \rho V(t)-\gamma^{-2} z^{\mathrm{T}}(t) z(t)+w^{\mathrm{T}}(t) w(t) .
$$

The third step is to show that $V$ does not increase during jumps. According to the proof of Theorem III.2 of [10], it holds that:

$$
\begin{aligned}
P_{i}(0)= & F_{21}(h) F_{11}^{-1}(h)+F_{11}^{-\mathrm{T}}(h)\left(P_{i h}+\right. \\
& P_{i h} \bar{S}\left(I-\bar{S}^{\mathrm{T}} P_{i h} \bar{S}\right)^{-1} \bar{S}^{\mathrm{T}} P_{i h} F_{11}^{-1}(h) .
\end{aligned}
$$

By applying Schur complement and S-procedure (see e.g. [2]), it follows from (23) that when there is an event on $u_{i}(t)$ the following holds:

$$
\begin{aligned}
\xi^{\mathrm{T}}(t) P_{i}(h) \xi(t) & \geq \xi^{\mathrm{T}}(t) J_{i}^{\mathrm{T}} P_{i^{+}}(0) J_{i} \xi(t) \\
& =\xi^{\mathrm{T}}\left(t^{+}\right) P_{i^{+}}(0) \xi\left(t^{+}\right) .
\end{aligned}
$$

Similarly, when there is no event on $u_{i}(t)$ the following holds:

$$
\begin{aligned}
\xi^{\mathrm{T}}(t) P_{i}(h) \xi(t) & \geq \xi^{\mathrm{T}}(t) J_{0}^{\mathrm{T}} P_{i^{+}}(0) J_{0} \xi(t) \\
& =\xi^{\mathrm{T}}\left(t^{+}\right) P_{i^{+}}(0) \xi\left(t^{+}\right) .
\end{aligned}
$$

The results of Theorem IV.2 guarantee that $V$ is a proper Lyapunov function and the system is GES with convergence rate $\rho$ when $w=0$ and has an $\mathcal{L}_{2}$-gain from $w$ to $z$ smaller than or equal to $\gamma$ which concludes our proof.

Theorem IV.2 solves the Problem III.1. The presented DPETC with asynchronous sampling approach ensures that only one transmission request is generated per sampling time $t_{k}$ while the pre-designed GES and $\mathcal{L}_{2}$-gain performance can still be guaranteed.

\section{NumericAl EXAMPLE}

In this section, we illustrate the presented theoretical results using a numerical example that describes the batch reactor system given in [18] with $E=\left[\begin{array}{cccc}1 & 0 & 0 & 0\end{array}\right]^{\mathrm{T}}$. Given the sampling interval $h=0.01 s$, the controller is obtained as:

$$
\left[\begin{array}{c|c}
A_{c} & B_{c} \\
\hline C_{c} & D_{c}
\end{array}\right]=\left[\begin{array}{cc|cc}
1 & 0 & 0 & 0.01 \\
0 & 1 & 0.01 & 0 \\
\hline-2 & 0 & 0 & -2 \\
0 & 8 & 5 & 0
\end{array}\right] .
$$



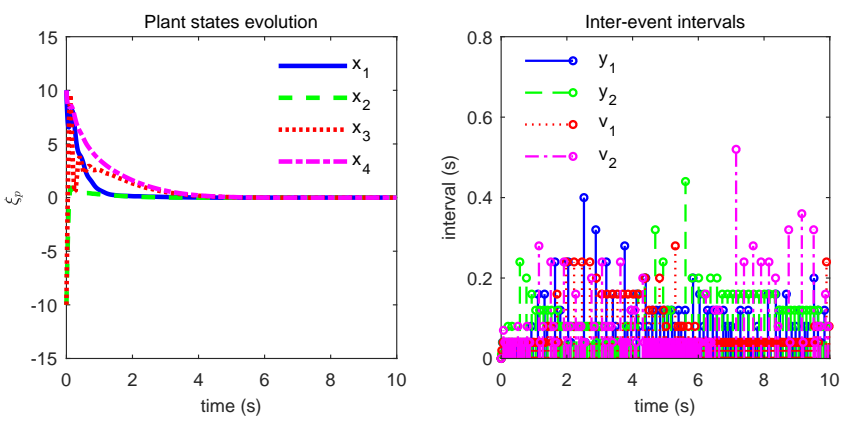

Fig. 4. Simulation result when $w=0$ : system state and inter-event intervals.
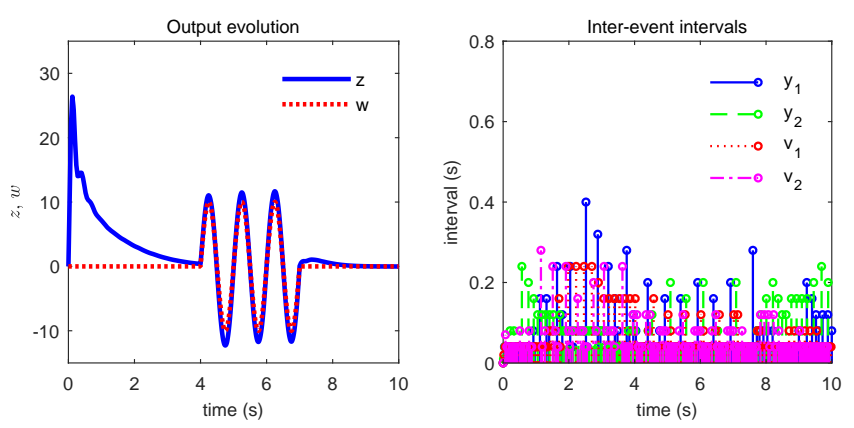

Fig. 5. Simulation result when $w(t)=10 \sin (2 \pi t), t=[4,7]$ : evolution of $z$ and $w$, and inter-event intervals.

Additionally, $\rho=0.01, \sigma_{i}=0.01, \forall i \in\left\{1, \cdots, n_{u}\right\}$, and $z(t)=\left[\begin{array}{llll}1 & 1 & 1 & 1\end{array}\right] \xi_{p}(t)+w(t)$. The initial state is given as $\xi_{p}(0)=\left[\begin{array}{llll}10 & -10 & -10 & 10\end{array}\right]^{\mathrm{T}}, \xi_{c}(0)=\mathbf{0}$, $\hat{y}(0)=C_{p} \xi_{p}(0)$, and $\hat{v}(0)=D_{c} C_{p} \xi_{p}(0)$.

Using the given parameters and by solving the LMIs (23), a feasible solution can be found for $\gamma=1.45$ (please note for this feasible solution, the $\mathcal{L}_{2}$-gain performance is less than or equal to $\gamma$ ). Further computation shows that the Assumption IV.1 is satisfied. Additionally, based on [10], we consider a disturbance that is represented as a sinusoidal type of noise. Fig. 4 and Fig. 5 show the simulation results of the system without and with disturbances. For both cases, we depict the evolution of the plant's states with respect to time and the time intervals between events for all outputs. The system states converge to an equilibrium point when $w=0$; and the $z$ is bounded when $w \in \mathcal{L}_{2}$, as designed. Also, the transmissions are distributed over the time-line and no bursty traffic patterns occur.

\section{CONCLUSION}

In this paper, we have proposed a decentralized periodic event-triggered control with asynchronous sampling scheme. Unlike the work in [7] and [10] that require the sensors to sample the system output synchronously at the same time, in our approach we apply the asynchronous sampling. This means that each sensor will sample the system output at its unique time which limits the number of samplings per each sampling time to one. Consequently, only one transmission request can be made which solves the problem of bursty traffic patterns in DPETC. This reduces the data load on the wireless network and provides a promising design for future TDMA MAC protocols. Future work will include the validation of proposed designs on real-world implementation (a higher dimensional system).

\section{REFERENCES}

[1] DP Borgers, VS Dolk, and WPMH Heemels. Riccati-based design of event-triggered controllers for linear systems with delays. IEEE Transactions on Automatic Control, 63(1):174-188, 2018.

[2] Stephen P Boyd, Laurent El Ghaoui, Eric Feron, and Venkataramanan Balakrishnan. Linear matrix inequalities in system and control theory, volume 15. SIAM, 1994.

[3] Dimos V Dimarogonas, Emilio Frazzoli, and Karl H Johansson. Distributed event-triggered control for multi-agent systems. IEEE Transactions on Automatic Control, 57(5):1291-1297, 2012.

[4] VS Dolk, DP Borgers, and WPMH Heemels. Output-based and decentralized dynamic event-triggered control with guaranteed $1_{-}$pgain performance and zeno-freeness. IEEE Transactions on Automatic Control, 62(1):34-49, 2017.

[5] MCF Donkers and WPMH Heemels. Output-based event-triggered control with guaranteed $\mathcal{L}_{\infty}$-gain and improved and decentralized event-triggering. IEEE Transactions on Automatic Control, 57(6):1362-1376, 2012.

[6] Fulvio Forni, Sergio Galeani, Dragan Nešić, and Luca Zaccarian. Event-triggered transmission for linear control over communication channels. Automatica, 50(2):490-498, 2014.

[7] Anqi Fu and Manuel Mazo, Jr. Decentralized periodic event-triggered control with quantization and asynchronous communication. Automatica, 94:294-299, 2018.

[8] Yanpeng Guan, Qing-Long Han, and Xiaohua Ge. On asynchronous event-triggered control of decentralized networked systems. Information Sciences, 425:127-139, 2018.

[9] Yanpeng Guan, Qing-Long Han, and Chen Peng. Decentralized event-triggered control for sampled-data systems with asynchronous sampling. In American Control Conference (ACC), 2013, pages 65656570. IEEE, 2013.

[10] WPMH Heemels, MCF Donkers, and Andrew R Teel. Periodic eventtriggered control for linear systems. IEEE Transactions on Automatic Control, 58(4):847-861, 2013.

[11] Sokratis Kartakis, Anqi Fu, Manuel Mazo Jr., and Julie A McCann. Communication schemes for centralized and decentralized eventtriggered control systems. IEEE Transactions on Control Systems Technology, 2017.

[12] Manuel Mazo Jr. and Ming Cao. Asynchronous decentralized eventtriggered control. Automatica, 50(12):3197-3203, 2014.

[13] Manuel Mazo Jr. and Paulo Tabuada. Decentralized event-triggered control over wireless sensor/actuator networks. IEEE Transactions on Automatic Control, 56(10):2456-2461, 2011.

[14] Alexandre Seuret. A novel stability analysis of linear systems under asynchronous samplings. Automatica, 48(1):177-182, 2012.

[15] Eduardo D Sontag. Input to state stability: Basic concepts and results. In Nonlinear and optimal control theory, pages 163-220. Springer, 2008.

[16] Paulo Tabuada. Event-triggered real-time scheduling of stabilizing control tasks. IEEE Transactions on Automatic Control, 52(9):16801685, 2007.

[17] Pavankumar Tallapragada and Nikhil Chopra. Decentralized eventtriggering for control of nonlinear systems. IEEE Transactions on Automatic Control, 59(12):3312-3324, 2014.

[18] Gregory C Walsh and Hong Ye. Scheduling of networked control systems. Control Systems, IEEE, 21(1):57-65, 2001.

[19] Xiaofeng Wang and Michael D Lemmon. Event-triggering in distributed networked control systems. IEEE Transactions on Automatic Control, 56(3):586-601, 2011.

[20] Xiaofeng Wang and Michael D Lemmon. On event design in eventtriggered feedback systems. Automatica, 47(10):2319-2322, 2011. 